\title{
OUTPUT DEAD BEAT CONTROL FOR A CLASS OF PLANAR POLYNOMIAL SYSTEMS*
}

\author{
D. NEŠIĆ* ${ }^{*}$ I. M. Y. MAREELS ${ }^{\dagger}$, G. BASTIN ${ }^{\ddagger}$, AND R. MAHONY*
}

\begin{abstract}
Output dead beat control for a class of non linear discrete time systems, which are described by a single input-output polynomial difference equation, is considered. The class of systems considered is restricted to systems with a two dimensional state space description. It is assumed that the highest degree with which the present input appears in the equation is odd. Necessary and sufficient conditions for the existence of output dead beat control and for the stability of the zero output constrained dynamics are presented. We also design a minimum time output dead beat control algorithm (feedback controller) which yields stable zero dynamics, whenever this is feasible. A number of interesting phenomena are discussed and illustrated by examples.
\end{abstract}

Key words. polynomial systems, dead beat, controllability

Subject classification: 93B05, 93B27, 93C55, 93D99

AMS subject classifications. 93B05, 93B27, 93C55, 93D99

1. Introduction. Linear dead beat control has received a great deal of attention in the last 30 years [16]. The discoveries in the area of linear dead beat control resulted in a better understanding of linear systems theory and a number of very successful applications. The fact that very often the dynamics of a plant can not successfully be modelled using linear time invariant equations, provide motivation for considering non linear dead beat control. Dead beat control or controllability for special classes of non linear systems has been addressed by many authors $[1,7,8,9,10,12,20,21]$. Nevertheless, a wealth of open questions remain to be explored.

Polynomial I-O systems of the form $y_{k+1}=f\left(y_{k}, \ldots, y_{k-s}, u_{k-t}, \ldots, u_{k}\right)$ are often used $[13,14,5]$ for system identification in black-box mode. $y, u$ and $k$ are respectively the output, input and time index. The function $f$ is a polynomial in all its arguments. This is an obvious generalisation of linear ARMA models. Although a number of applications of I-O polynomial systems have been reported, e.g. [5, 14], their control properties are not well understood.

In this paper we consider a class of I-O polynomial systems of the following form:

$$
y_{k+1}=f\left(y_{k}, u_{k-1}, u_{k}\right)
$$

We assume throughout the paper that the highest exponent of the argument $u_{k}$ in the polynomial $f$ is an odd integer. An application of this class of systems can be found in [5] where a subsystem of a radiator and fan is identified in this form.

The control question that we are interested in is minimum time output dead beat regulation. In particular, we want to design a control law of the form:

$$
u_{k}=c\left(y_{k}, u_{k-1}\right)
$$

such that $y_{k}=0, \forall k \geq t$, for some integer $t$ and such that the constrained dynamics ${ }^{1}$

* This work is supported by the Cooperative Research Centre for Adaptive and Robust Systems, Australia

* Department of Systems Engineering, RSISE, ANU, Canberra, ACT 0200, AUSTRALIA. e-mail address: dragan.nesic@anu.edu.au, tel. - int $+61+6+2492456$, fax. - int $+61+6+2492698$

$\dagger$ Engineering Department, FEIT, ANU, Canberra, ACT 0200, AUSTRALIA.

$\ddagger$ CESAME, Batiment Euler, Avenue G.Lemaitre 4, 1348 Louvain-La-Neuve, Belgium. This work was done while the author was visiting the Australian National University 
defined by:

$$
\begin{aligned}
0 & =f\left(0, u_{k-1}, u_{k}\right) \\
u_{k} & =c\left(0, u_{k-1}\right)
\end{aligned}
$$

are stable in a sense to be specified later. The paper deals with two questions: output dead beat controllability and stability of constrained dynamics for (1).

Some pioneering work on controllability for a class of discrete time bilinear systems can be found in [11]. Papers [7, 8, 12] provide complete conditions for controllability for the same class of systems. Invariance of the control independent set was investigated in [11]. We show that a new notion of strongly invariant sets, first introduced in [20], is crucial for output dead beat controllability of (1). We take a similar approach as in [20], where dead beat controllability of scalar polynomial systems is considered. The output controllability result of this paper can be viewed as a generalisation of some results on odd systems in [20]. In the conference version [21] of this paper, we provided the output controllability test for (1). However, the design of a feasible dead beat controller and stability of constrained dynamics are analysed in the sequel.

Output dead beat control of recursive nonlinear systems was investigated in [1, 3]. Existence of constrained dynamics together with a number of interesting phenomena were studied. The considered class of systems was, however, large and results are consequently weak. The notion of criterion of choice is introduced in the context of stability of constrained dynamics in $[1,3]$. This notion is also important in our discussions. Stability of one dimensional explicit constrained dynamics $u_{k}=f\left(u_{k-1}\right)$ was investigated $[2,3]$. Our paper extends these results to the case of implicitly defined polynomial dynamics (3) and we present necessary and sufficient conditions of the existence of a criterion of choice that leads to stable constrained dynamics. We point out that the stability of an interval that we consider was investigated in [3] and in [15], where this property is referred to as "permanence". In [15], global stability properties of a number of nonlinear explicit systems of the form $y_{k+1}=F\left(y_{k}, \ldots, y_{k-s}\right)$ was investigated. We, however, consider the implicit difference equation in (3). We emphasize that the notion of constrained dynamics considered here differs from the concept of zero dynamics introduced in [17, 18]. Moreover, the notion of zero dynamics $[17,18]$ appears not to be sufficiently general to be applicable to the stabilising dead beat control problem considered here.

This paper provides an explicit test for verifying the existence of an output dead beat control law which yields stable constrained dynamics for the system of the form (1). Furthermore, a constructive design method is provided to find any such feedback law. A purpose of this paper is to show the difficulties that one may face when tackling output dead beat control problem for the simple class of I-O polynomial systems (1) and to present a number of interesting phenomena.

The paper is organised as follows. In Section 2 we present some notation and in Section 3 we define the problem and the class of systems that we consider. The question of the existence of dead beat control is addressed in Section 4. Sections 5 and 6 are respectively dedicated to the stability of constrained dynamics and a method to check the existence of a dead beat control law which yields stable constrained dynamics. The modified dead beat control law which zeroes the output in minimum

\footnotetext{
1 The definition of stable zero output constrained dynamics that we analyse is more general than the usual definition of zero dynamics found in literature [17]. To make the distinction more obvious we refer to our definition as constrained dynamics and to the definition in [17] as zero dynamics.
} 
time and also yields stable constrained dynamics is then presented in Section 7 . In Section 8, we present several examples which illustrate our methods. The summary and conclusion are given in the last section.

2. Mathematical preliminaries. We use the standard definitions of rings and fields [6]. We work over the field of real numbers which is denoted as $\Re$. $\Re^{n}$ is a set of all $n$-tuples of elements of $\Re$, where $n$ is a non negative integer. The ring of polynomials in $n$ variables over the real field $\Re$ is denoted as $\Re\left[x_{1}, x_{2}, \ldots, x_{n}\right]$. Let $f_{1}, f_{2}, \ldots, f_{s}$ be polynomials in $\Re\left[x_{1}, x_{2}, \ldots, x_{n}\right]$. Then we define

$$
V\left(f_{1}, f_{2}, \ldots, f_{s}\right)=\left\{\left(a_{1}, a_{2}, \ldots, a_{n}\right) \in \Re^{n}: f_{i}\left(a_{1}, a_{2}, \ldots, a_{n}\right)=0 \text { for all } 1 \leq \mathrm{i} \leq \mathrm{s}\right\} .
$$

We call $V\left(f_{1}, f_{2}, \ldots, f_{s}\right)$ the real algebraic set or real variety defined by $f_{1}, f_{2}, \ldots, f_{s}$. Since the defining polynomials of a real variety are often clear from the context, it is often denoted simply as $V$.

DEFINITION 1. A real variety $V \subset \Re^{n}$ is irreducible if whenever $V$ is written in the form $V=V_{1} \cup V_{2}$, where $V_{1}$ and $V_{2}$ are real varieties then either $V_{1}=V$ or $V_{2}=V$. [6, pg. 196].

TheOREM 2.1. [6, pg. 202] Let $V \subset \Re^{n}$ be a real variety. Then $V$ can be written as a finite union of irreducible varieties $V=V_{1} \cup V_{2} \cup \ldots \cup V_{m}$ where each $V_{i}$ is an irreducible variety.

Let $f, g \in \Re\left[x_{1}, x_{2}, \ldots, x_{n}\right] . f \mid g$ means that $g$ divides $f$, that is, there exists a polynomial $h \in \Re\left[x_{1}, x_{2}, \ldots, x_{n}\right]$ such that $f=h g . f \equiv g \mid h$ means that $f$ is divisible by $h$ modulo $g$, that is, given polynomials $h$ and $g, \operatorname{deg}(g)<\operatorname{deg}(f)$ there exists a polynomial $h_{1} \in \Re\left[x_{1}, x_{2}, \ldots, x_{n}\right]$ such that $f=h_{1} h+g$. Also, $f \backslash \not g$ and $f \equiv g \backslash h$ denotes respectively that $f$ is not divisible by $g$ and $f$ is not divisible by $h$ modulo $g$.

We say that a variety $V \subset \Re^{2}$ has Special Form if

$$
V=\left\{(y, v) \in \Re^{2}: y-\sum_{i=0}^{n-1} b_{i} v^{i}=0, b_{i} \in \Re, i=0,1, \ldots, n-1\right\} .
$$

Varieties of Special Form are irreducible because they can be parametrized by polynomials [6, pg. 197].

3. Definition of the system. We consider systems described by the following recursive input-output polynomial equation:

$$
y_{k+1}=f\left(y_{k}, u_{k-1}, u_{k}\right)
$$

where $y_{k}$ is the output of the system at time $k$ and $u_{k}$ is the input to the system at time $k$. The function $f$ is a polynomial, $f \in \Re[y, v, u]$. We assume that the highest exponent of $u$ in $f(y, v, u)$ is an odd integer. A system (4) with this property is referred to as an odd system.

It is always possible to rewrite (4) in the following form:

$$
y_{k+1}=g_{n}\left(y_{k}, u_{k-1}\right) u_{k}^{n}+g_{n-1}\left(y_{k}, u_{k-1}\right) u_{k}^{n-1}+\ldots+g_{0}\left(y_{k}, u_{k-1}\right)
$$

where $g_{n} \not \equiv 0$ and $n$ is an odd positive integer.

Assumption 1. Constrained dynamics are defined:

$$
\forall v \in \Re, \exists u \in \Re \text { such that } f(0, v, u)=0
$$


A sequence of controls is denoted as

$$
\mathcal{U}=\left\{u_{0}, u_{1}, \ldots\right\}
$$

The truncation to a sequence of length $p+1$ is denoted as $\mathcal{U}_{p}=\left\{u_{0}, u_{1}, \ldots, u_{p}\right\}$. The composition of the function $f$ in equation (4) under the action of a control sequence $\mathcal{U}_{p}$ which starts from $\left(y_{0}, u_{-1}\right) \in \Re^{2}$ is denoted as

$$
f^{\mathcal{U}_{p}}\left(y_{0}, u_{-1}\right)=\underbrace{f(f(\ldots f(f}_{p \text { times }}\left(y_{0}, u_{-1}, u_{0}\right), u_{0}, u_{1}), \ldots, u_{p-1}, u_{p})
$$

Obviously $y_{p+1}=f^{\mathcal{U}_{p}}\left(y_{0}, u_{-1}\right)$ is the output at time $p+1$, given the starting point $\left(y_{0}, u-1\right)$ and the input $\mathcal{U}_{p}$.

We can introduce the state variables $x_{1}(k)=y_{k}$ and $x_{2}(k)=u_{k-1}$ and write accordingly the model in state space format. In the sequel, we refer to $\left(y_{0}, u_{-1}\right) \in \Re^{2}$ as an initial state although we work with the input output equation (4).

We are interested in output dead beat control:

Definition 2. The system (4) is output dead beat controllable if for every $\left(y_{0}, u_{-1}\right) \in \Re^{2}$ there is a sequence $\mathcal{U}=\left\{u_{0}, u_{1}, \ldots\right\}$ such that the output of the system (4) is driven to zero in finite time, that is, $y_{k}=0, \forall k \geq t$, where $t$ is a non negative integer.

Definition 3. A feedback controller, given by $u_{k}=c\left(y_{k}, u_{k-1}\right)$, is an output dead beat controller if there exists a positive integer $P$ such that $\forall\left(y_{0}, u_{-1}\right) \in \Re^{2}$, and $k \geq P$, we have $y_{k}=0$, where $y_{k+1}=f\left(y_{k}, u_{k-1}, c\left(y_{k}, u_{k-1}\right)\right)=0$.

Because of Assumption 1, we can split the dead beat control problem into two parts. Indeed, the control sequence $\mathcal{U}$ in Definition 2 may be split into two parts. $\mathcal{U}_{t}$ is the part of the sequence $\mathcal{U}$ that transfers the output to the origin and $\left\{u_{t+1}, \ldots\right\}$ the part which keeps the output at the origin. Section 4 is concerned with the existence of the sequence $\mathcal{U}_{t}$, which naturally leads to the construction of an (feedback) output dead beat controller. In Section 5 we consider the properties of the obtained control laws, which settles the usefulness of the approach.

4. Output dead beat controllability of recursive polynomial systems. In this section, we consider when it is possible to transfer the output of the system (4) to the origin in finite time, starting from an arbitrary initial state $\left(y_{0}, u_{-1}\right) \in \Re^{2}$. The following definition is used in the sequel:

Definition 4. The one step reachable set from an initial state $\left(y_{0}, u_{-1}\right) \in \Re^{2}$ is defined as

$$
V_{r}\left(y_{0}, u_{-1}\right)=\left\{(y, u) \in \Re^{2}: y-f\left(y_{0}, u_{-1}, u\right)=0\right\}
$$

We also define the projection of the one step reachable set onto the first coordinate axis as:

$$
\Pi V_{r}\left(y_{0}, u_{-1}\right)=\left\{y \in \Re: \exists v \in \Re:(y, v) \in V_{r}\left(y_{0}, u_{-1}\right)\right\}
$$

and call it the set of one step reachable outputs.

Observe that the one step reachable set is a real variety and it has Special Form for any initial state in $\Re^{2}$. Moreover, since the systems is odd, the only states from which it may not be possible to zero the output in one step belong to the real variety $V_{C}$ defined by

$$
V_{C}=\left\{(y, v) \in \Re^{2}: g_{n}(y, v)=0\right\}
$$


Notice that $\operatorname{dim} V_{C}<2$.

Definition 5 . The variety $V_{C}$ given by $(7)$ is called the critical variety.

DEFinition 6. The number of varieties of Special Form that are contained in $V_{C}$ is denoted by $N$.

Let $V$ and $W$ be varieties. We introduce notation:

$$
V \stackrel{f}{\rightarrow} W
$$

to denote that $V_{r}\left(y_{0}, u_{-1}\right)=W, \forall\left(y_{0}, u_{-1}\right) \in V$. It should be emphasised that the equation (8) means that the one step reachable set from any initial state in $V$ is equal to $W$.

Definition 7. A set $V_{I_{j}} \subseteq V_{C}$ is invariant if

$$
\forall(y, v) \in V_{I_{j}}, V_{r}(y, v) \subseteq V_{I_{j}}
$$

The union of all invariant sets $V_{I}=\cup_{j} V_{I_{j}}$ is called the maximal invariant set.

Definition 8. A subset $W_{I_{j}}$ of the variety $V_{C}$ is strongly invariant if it is invariant and $\forall\left(y_{0}, u_{-1}\right) \in W_{I_{j}}$ there exists an integer $t \geq 0, t=t\left(y_{0}, u_{-1}\right)$ and a sequence of controls $\mathcal{U}_{t}=\left\{u_{0}, u_{1}, \ldots, u_{t}\right\}$ which yields $\left(y_{t+1}, u_{t}\right)=\left(y_{0}, u_{-1}\right)$ where $y_{t+1}=f^{\mathcal{U}_{t}}\left(y_{0}, u_{-1}\right)$. The union of all strongly invariant sets $W_{I}=\cup_{j} W_{I_{j}}$ is called the maximal strongly invariant set.

Definition 9. The number of varieties of Special Form that are contained in the maximal strongly invariant set $W_{I}$ of $V_{C}$ is denoted by $L$.

The propositions below indicate some important properties of the maximal invariant and strongly invariant sets.

Proposition 4.1. The maximal strongly invariant set can be decomposed into finitely many strongly invariant subsets $W_{I_{j}}$, each of which can be decomposed into finitely many varieties of Special Form $W_{i}$ :

$W_{I}=\underbrace{W_{1} \cup \ldots \cup W_{L_{1}}}_{W_{I_{1}}} \cup \underbrace{W_{L_{1}+1} \ldots \cup W_{L_{1}+L_{2}}}_{W_{I_{2}}} \cup \ldots \cup \underbrace{\cup W_{L_{1}+\ldots+L_{p-1}+1} \cup \ldots \cup W_{L_{1}+\ldots+L_{p}}}_{W_{I_{p}}}$

where $L_{1}+L_{2}+\ldots+L_{p}=L$. Therefore, the maximal strongly invariant set is itself a variety.

Sketch of the proof: We prove this proposition in four steps. Since $V_{r}\left(y_{0}, u_{-1}\right)$ is of Special Form for any $\left(y_{0}, u_{-1}\right) \in V_{C}$, at least one variety of Special Form $W_{1}$ belongs to the maximal strongly invariant subset $W_{I}$. Then we can show that in order to have invariance one step reachable sets from any initial state in $W_{1}$ must coincide, that is $V_{r}\left(y_{1}, v_{1}\right)=V_{r}\left(y_{2}, v_{2}\right), \forall\left(y_{1}, v_{1}\right),\left(y_{2}, v_{2}\right) \in W_{1}$. Therefore, we show that one can write $V_{r}(y, v)=W_{2}, \forall(y, v) \in W_{1}$, where $W_{2}$ is a variety of Special Form which is a subset of $V_{C}$. After this, we show that the union $W_{1} \cup W_{2} \cup \ldots \cup W_{L}$ is a subset of $W_{I}$. Finally, it is proved that the union $W_{1} \cup W_{2} \cup \ldots \cup W_{L}$ is equal to $W_{I}$, and the partition into smaller strongly invariant sets follows easily. For a more detailed proof see [21]. Q.E.D.

Proofs of the propositions below hinge on the proof of Proposition 4.1 (see [21]).

Proposition 4.2. Any invariant subset $V_{I_{j}}$ of the critical variety $V_{C}$ contains a strongly invariant subset $W_{I_{j}}$.

Proposition 4.3. Any initial state that belongs to an invariant subset $V_{I_{j}}$ of the critical variety $V_{C}$ is transferred to a strongly invariant subset $W_{I_{j}}$ (which is a subset of $\left.V_{I_{j}}\right)$ in finite time. 
Proposition 4.4. Any $\left(y_{0}, u_{-1}\right) \in V_{C}-V_{I}$ can be mapped to $\Re^{2}-V_{C}$ in at most $N-L+1$ time steps (see Definitions 6 and 9 ).

Comment 1. An immediate consequence of Proposition 4.4 is that if $V_{I}=\emptyset$ any initial state in $V_{C}$ can be mapped to $\Re^{2}-V_{C}$ in at most $N+1$ time steps and hence the output can be zeroed in at most $N+2$ steps (see Definition 6 ).

Proposition 4.5. Consider the system (4). The critical variety $V_{C}(7)$ contains a strongly invariant subset if and only if there exist polynomials $y-\sum_{i=0}^{n-1} b_{i}^{p} v^{i}, b_{i}^{p} \in$ $\Re, \quad p=1,2, \ldots, B, B \leq L \leq N$ such that

$$
\begin{gathered}
g_{n}(y, v) \mid\left(y-\sum_{i=0}^{n-1} b_{i}^{p} v^{i}\right), \forall p=1,2, \ldots, B \\
g_{i}(y, v) \equiv b_{i}^{p+1} \mid\left(y-\sum_{i=0}^{n-1} b_{i}^{p} v^{i}\right), \forall p=1,2, \ldots, B-1, \forall i=1, \ldots, n-1, \quad \text { and } \\
g_{i}(y, v) \equiv b_{i}^{1} \mid\left(y-\sum_{i=0}^{n-1} b_{i}^{B} v^{i}\right), \forall i=1, \ldots, n-1 \quad \square
\end{gathered}
$$

The above properties of invariant subsets of $V_{C}$, lead to necessary and sufficient conditions for output dead beat controllability for the class of odd systems (4).

THEOREM 4.6. The odd system (4) is output dead beat controllable if and only if either the maximal invariant set $V_{I}=\emptyset$ or if $V_{I} \neq \emptyset$, then all irreducible components (varieties) $W_{i}, i=1,2, \ldots, L$ of the maximal strongly invariant set $W_{I}$ intersect the line $y=0$.

Sketch of the proof: The whole state space can be partitioned as $W_{I} \cup\left(V_{I}-\right.$ $\left.W_{I}\right) \cup\left(V_{C}-V_{I}\right) \cup\left(\Re^{2}-V_{C}\right)$. Propositions 4.1, 4.2, 4.3, 4.4, together with the fact that any state in $V_{C}-V_{I}$ can be mapped to $\Re^{2}-V_{C}$, give a characterisation of all possible behaviours. Q.E.D.

Comment 2. It is easily verified that the conditions under which the critical variety $V_{C}$ may contain invariant subsets (they are given in Proposition 4.5) are clearly not generic.

Comment 3. It is important to notice that Theorem 4.6 provides conditions for output controllability to the origin. If we want to check output controllability to some other point $y^{*} \neq 0$ then all irreducible components (varieties) $W_{i}$ of the maximal strongly invariant set $W_{I}$ should intersect the line $y=y^{*}$.

5. Stability of Constrained Dynamics. We examine in this section properties of the control law which keeps the output of the system at zero after the output was zeroed. We extend Theorem 6.2 [3] to the class of polynomial implicitly defined systems. This theorem gives necessary and sufficient conditions for the global stability of an invariant interval for the class of explicit constrained dynamics defined by $u_{k}=$ $g\left(u_{k-1}\right)$ with $g$ continuous. We consider implicitly defined polynomial systems. The equation that defines the behaviour of the system is given below:

$$
f\left(0, u_{k-1}, u_{k}\right)=0 .
$$

It was noticed in [1] that the properties of the control law that keeps the output at zero depends on the rule used to determine which particular solution from among 
the possible alternatives $u_{k}$, satisfying (10), is used for any given $u_{k-1}$. This rule is referred to as a criterion of choice. If we have several control actions that satisfy the constraint (10) at our disposal, it is very important to apply "the most appropriate one".

In this section we define what we mean by stable constrained dynamics and find conditions which guarantee the existence of a "good" criterion of choice, i.e. one that leads to stable constrained dynamics. Now we give definitions for the concepts that we need in our developments.

Definition 10. A criterion of choice is a single valued function $c: \Re \rightarrow \Re$ (denoted also as $u_{k}=c\left(u_{k-1}\right)$ ) such that

$$
f(0, v, c(v))=0, \quad \forall v \in \Re .
$$

Definition 11. Consider a criterion of choice $c$ (Definition 10). A bounded interval $A \subset \Re$ is invariant under mapping $c$ if $c(A) \subseteq A$.

Definition 12. Let $A \subset \Re$ be a bounded interval invariant under mapping $c$. Then:

1. $A$ is called stable if $\forall E \subseteq \Re, A \subset E, \exists K(E)>0$ such that $\forall u_{k-1} \in E$ we have $\sup _{u_{k-1} \in E}\left|c\left(u_{k-1}\right)\right| \leq K(E)<\infty$.

2. $A$ is called attractive if $\forall \Delta>0, \forall u_{-1}, \exists T=T\left(\Delta, u_{-1}\right)$ such that $\inf _{x \in A}, \mid x-$ $u_{k} \mid<\Delta, \forall k>T$.

3. $A$ is called asymptotically stable if 1 and 2 hold

DEFINITION 13. Implicitly defined constrained dynamics (equation (10)) are called stable if there exists a criterion of choice $c$ such that there is a bounded interval $A$ invariant under mapping $c$ which is asymptotically stable.

We emphasize that the present notion of stability is more general than allowed for in $[17,18]$, where only stability of equilibria is considered. Notice also that we consider a global stability property.

We now cite Theorem 6.2 from [3] which is used in the proof of the main result.

Theorem 5.1. [3] Consider the map $g: \mathbf{D} \rightarrow \mathbf{D}, \mathbf{D} \subset \Re$. Let $\mathcal{A} \triangleq[a, b] \subset \Re$ such that:

1. $\mathbf{D} \cap \mathcal{A}$ is invariant under $g: g(\mathbf{D} \cap \mathcal{A}) \subset \mathbf{D} \cap \mathcal{A}$

2. $(\Re-] a, b[) \subset \mathbf{D}$

3. $g$ is continuous on $(\Re-] a, b[)$

Then $\mathcal{A}$ is globally attracting interval of the iterative map $u(k+1)=g(u(k))$ if and only if the following conditions hold:

$$
\begin{array}{lll}
\forall x<a & g(x)>x \\
\forall x>b & g(x)<x \\
\forall x<a & \text { such that } \exists(x, z) \in G_{R}^{-1} & g(x)<z \\
\forall x>b & \text { such that } \exists(x, z) \in G_{L}^{-1} & g(x)>z
\end{array}
$$

The domain $\mathbf{D}$ represents the domain of definition of constrained dynamics. Other symbols used in the statement of Theorem 5.1 are given below:

$$
\begin{aligned}
& G=\{(x, g(x)): x \in \Re-[a, b]\}, \quad G_{L}=\{(x, g(x)) \in G: x<a\}, \\
& G_{R}=\{(x, g(x)) \in G: x>b\}, \quad G_{L}^{-1}=\left\{(g(x), x):(x, g(x)) \in G_{L}\right\}, \\
& G_{R}^{-1}=\left\{(g(x), x):(x, g(x)) \in G_{R}\right\}
\end{aligned}
$$


Comment 4. In our case the domain of definition of constrained dynamics is the whole real line, that is $\mathbf{D}=\Re$. Therefore, Condition 2 of Theorem 5.1 does not need to be verified.

Given $T \geq 0$ a real number, the following sets will be used in the sequel:

$$
S_{1}=\left\{(v, u) \in \Re^{2}: v<-T\right\}, \quad S_{2}=\left\{(v, u) \in \Re^{2}: v>T\right\}
$$

A very important feature of polynomial systems which is crucial for the stability of constrained dynamics is given in the theorem below.

THEOREM 5.2. Consider the real variety $V_{z}$ defined by

$$
V_{z}=\left\{(v, u) \in \Re^{2}: f(0, v, u)=0\right\}
$$

There exists $T \geq 0$ such that there are constant numbers $n_{1}$ and $n_{2}$ of continuous branches ${ }^{2}$ of variety $V_{z}$ on sets $S_{1}$ and $S_{2}$ (13).

Proof of Theorem 5.2: Sturm sequences can be used in order to check the exact number of distinct real roots of a univariate polynomial on any interval $[a, b]$, including ] $-\infty,+\infty\left[\right.$ [4]. We will regard $u_{k-1}$ as a parameter and for any fixed $u_{k-1}$ we can find the number of distinct real roots $u_{k}$ to $(10)$. In other words, we can find the exact number of real roots to $(10)$ on vertical lines $u_{k-1}=$ const..

Consider the Sturm sequence of $f(0, v, u)$. It has the form:

$$
\begin{aligned}
& A_{n}^{0}(v) u^{n}+\ldots+A_{0}^{0}(v) \\
& A_{n-1}^{1}(v) u^{n-1}+\ldots+A_{0}^{1}(v) \\
& \ldots \\
& A_{0}^{n}(v)
\end{aligned}
$$

The leading coefficient functions are rational functions in $v$. It turns out that for the number of real solutions $u$ to (10) for a fixed value of the parameter $v$, only the leading coefficient functions are important. Actually, the signs of these functions determine the number of real roots and since they are rational functions, we can find a set of the form $]-\infty,-D_{1}[\cup] D_{1},+\infty$ [ on which their signs do not change. The modified division algorithm which is used to determine the sequence (15) yields a special form of the leading coefficients in the Sturm sequence. Namely, the denominator of $A_{n-j+1}^{j+1}(v)$ has the same roots as the numerator of $A_{n-j}^{j}(v), \forall j>1$. Also, $A_{n}^{0}=g_{n}(0, v)$ and $A_{n-1}^{1}=n \partial / \partial v\left[g_{n}(0, v)\right]$ are polynomials. Consequently, the set on which the $A_{n-j}^{j}(v)$ do not change signs can be determined considering the equations $A_{n-j}^{j}(v)=0, \forall j=$ $0, \ldots, n$. We introduce the following set:

$$
\mathcal{D}_{1}=\left\{v \in \Re: A_{n-j}^{j}(v)=0, \text { for some } j=0, \ldots, n\right\}
$$

Denote as $D_{1}$ the following number:

$$
D_{1}=\sup _{v \in \mathcal{D}_{1}}|v|
$$

It follows that on the set $]-\infty,-D_{1}[\cup] D_{1},+\infty[$ all the leading coefficient functions do not change their signs. Therefore, there is a constant number of real roots $u_{k}$ for

\footnotetext{
2 The term "branch of $V_{z}$ " that we use corresponds to parts of irreducible varieties (curves) from which the variety $V_{z}$ is composed $[4,6]$ that belong to sets $S_{1}$ and $S_{2}$.
} 
every $\left.u_{k-1} \in\right]-\infty,-D_{1}\left[\right.$ and $\left.u_{k-1} \in\right] D_{1},+\infty[$ to equation (10). We can also say that there exist a constant number of continuous branches of $V_{z}$ on sets $]-\infty,-D_{1}[\times \Re$ and $] D_{1},+\infty[\times \Re$. This follows from the theorem on the continuity of real roots [4, pg. 38]. Since $g_{n}(0, v) \neq 0\left(g_{n}=A_{n}^{0}\right)$ for $\left.v \in\right]-\infty,-D_{1}[\cup] D_{1},+\infty[$ and since there is a constant number of complex roots all the conditions of the theorem are satisfied. Q.E.D.

Comment 5. Theorem 5.2 states that it is possible to find an interval $\left[-D_{1}, D_{1}\right]$ inside which all bifurcations of the variety $V_{z}$ occur. Moreover, from the theorem on the continuity of roots $[4, \mathrm{pg}$. 38] we see that all intersections between branches of the variety $V_{z}$ occur inside the same interval.

LEMma 5.3. A necessary condition for the existence of stable constrained dynamics is

$$
\left.\sup _{|v|<K} \inf _{(v, u) \in V_{z}}|u|<+\infty, \quad \forall K \in\right] 0,+\infty[
$$

Proof of Lemma 5.3: Suppose that there exists a criterion of choice $c$ which yields stable constrained dynamics. Suppose that there exists $v=u_{k-1}^{*}$ which belongs to the invariant interval such that all branches of the variety $V_{z}$ have a vertical asymptote at $v=u_{k-1}^{*}$. In other words, the condition of Lemma 5.3 is not satisfied for any neighbourhood of the origin that contains $u_{k-1}^{*}$. It is then obvious that the invariant interval must have one of the following forms: $]-\infty,+\infty[,[K,+\infty[$ or $]-\infty, K]$ and we have a contradiction since neither of these intervals is bounded. Suppose now that $u_{k-1}^{*}$ does not belong to the invariant interval. In this case, constrained dynamics can not be stable in the sense of Definition 13 because for $u_{k-1}$ such that $u_{k-1} \rightarrow u_{k-1}^{*}$ we have that $\left|u_{k}\right| \rightarrow+\infty$, so we again obtain a contradiction. Q.E.D.

Now we can give definitions of maximal and minimal branches of the variety $V_{z}$.

Definition 14 . Consider the variety $V_{z}$ on sets $S_{1}$ and $S_{2}$. The maximal branch of $V_{z}$ in $S_{2}$ is given by:

$$
V_{M}^{S_{2}}=\{(v, u): v \in] T,+\infty\left[, u=\max _{(v, y) \in\left(V_{z} \cap S_{2}\right), y<v} y\right\}
$$

The minimal branch of $V_{z}$ in $S_{1}$ is such that:

$$
V_{m}^{S_{1}}=\{(v, u): v \in]-\infty,-T\left[, u=\min _{(v, y) \in\left(V_{z} \cap S_{1}\right), y>v} y\right\} .
$$

In other words, the maximal branch is the closest branch of $V_{z}$ to the bisector $u=v$, which is below the bisector (on the set $S_{2}$ ). Notice that minimal and maximal branches are well defined parts of irreducible varieties of $V_{z}$, following from the theorem on continuity of roots [4] and Bezout's theorem [6]. Bezout's theorem says that we can find a set $\left[-D_{3}, D_{3}\right] \times \Re$ inside which all intersections between the variety $V_{z}$ and the bisector $u=v$ occur. Also notice that if there are no branches in $S_{2}$ that are below the bisector $u=v$, then by definition $V_{M}^{S_{2}}=\emptyset$.

Comment 6. Suppose that we can find a criterion of choice such that outside a bounded interval $[-T, T]$ all orbits are bounded, converge to the interval and enter it in finite time from any given $u_{-1}$. Then it is easy to show that when Lemma 5.3 holds there exists an interval (perhaps larger than $[-T, T]$ but bounded) such that it is invariant and stable. Consequently, we will concentrate only on the existence of a bounded stable interval and Lemma 5.3 guarantees that we can always have a criterion of choice for all $u_{-1} \in[-T, T]$ which renders the interval invariant. 
Now we can state the main result.

THEOREM 5.4. Implicitly defined constrained dynamics (10) are stable if and only if the mapping $u_{k}=g\left(u_{k-1}\right)$ defined as

$$
u_{k}= \begin{cases}y & \text { such that }\left(u_{k-1}, y\right) \in V_{m}^{S_{1}} \text { if } u_{k-1}<-T \\ y & \text { such that }\left(u_{k-1}, y\right) \in V_{M}^{S_{2}} \text { if } u_{k-1}>T \\ y & \text { such that }\left(u_{k-1}, y\right) \in V_{z} \text { if } u_{k-1} \in[-T, T] \text { and } \\ & y \text { has the smallest absolute value }\end{cases}
$$

satisfies equations (12) of Theorem 5.1 and Lemma 5.3 holds.

Proof of Theorem 5.4:

Sufficiency: Consider the criterion of choice (18). It is obvious that all the conditions of Theorem 5.1 are satisfied and this criterion yields stable constrained dynamics.

Necessity: We only have to show that the conditions (12) are necessary for stable constrained dynamics. We can find a set inside which all intersections between the variety $V_{z}$ and the bisector $u=v$ occur and denote it as $\left[-D_{3}, D_{3}\right] \times \Re$. Moreover, we can find another set inside which all the intersections between $V_{z}$ and $V_{z}^{-1}=\{(v, u) \in$ $\left.\Re^{2}: f(0, u, v)=0\right\}$ occur (modulo common components which may have infinitely many common points) and denote it as $\left[-D_{2}, D_{2}\right] \times \Re$. All the subsequent arguments are given for the sets $S_{1}$ and $S_{2}$ defined by the number $T=\max \left[D_{1}, D_{2}, D_{3}\right]$. Sets $S_{1}$ and $S_{2}$ (13) defined in this way obviously have the property that (modulo common components) there are no intersections between $V_{z}$ and $V_{z}^{-1}$ on the sets, there are no bifurcations of the variety $V_{z}$ on the sets and, finally, minimal and maximal branches $V_{m}^{S_{1}}$ and $V_{M}^{S_{2}}$ are either parts of continuous curves or they are empty sets.

Suppose that the constrained dynamics are stable and that the first condition in (12) is not satisfied. Since $V_{m}^{S_{1}}=\emptyset$, all branches are below the bisector $u=v$ and as a consequence we have that $u_{k} \rightarrow-\infty$ as $\left.\left.k \rightarrow \infty, \forall u_{-1} \in\right]-\infty,-T\right]$. A similar situation happens when the second condition (12) is not satisfied and therefore the first two conditions in (12) are necessary to ensure stability of the constrained dynamics. In other words, a necessary condition for the stability of the implicitly defined constrained dynamics (10) is that $V_{m}^{S_{1}} \neq \emptyset$ and $V_{M}^{S_{2}} \neq \emptyset$.

Consider now what happens if the third condition in (12) is not satisfied. Since all branches of $V_{z}$ in $S_{2}$ are above $V_{M}^{S_{2}}$, all their inverses will lay on the left hand side (or below) of $\left(V_{M}^{S_{2}}\right)^{-1}$. Thus, we suppose that no branch of $V_{z}^{-1}$ satisfies the third condition in (12). Moreover, if we use pieces of branches of $V_{z}$ to construct a piecewise continuous one to one function and use the modified Theorem 5.1 [3] we can see that no such function would satisfy the conditions of Theorem 5.1. Therefore, there does not exist a criterion of choice which yields stable constrained dynamics. The contradiction completes the proof. The last two conditions are symmetric and they are either both satisfied or not. Q.E.D.

6. An Algebraic Test for Stability of Constrained Dynamics. We now present a method to check the conditions of Theorem 5.4. First, we provide a means of verifying the conditions of Lemma 5.3.

We write the function (10) as

$$
f(0, v, u)=g_{n}(0, v) u^{n}+\ldots+g_{0}(0, v)
$$

The only critical points that we have to check are the ones for which the leading coefficient $g_{n}(0, v)(19)$ vanishes [4, pg. 10, pg. 39]. Therefore, the first step is to find 
all real solutions $v$ to $g_{n}(0, v)=0$. It is then necessary to check whether

$$
f(0, v, u)=0
$$

has real roots $u$, for all critical values of $v$. We define the following sets:

$$
\begin{aligned}
\mathcal{A} & =\left\{v: g_{n}(0, v)=0\right\} \\
\mathcal{B}(v) & =\{u \in \Re: f(0, v, u)=0\}, v \in \mathcal{A} \\
\mathcal{C} & =\{(v, u): v \in \mathcal{A}, u \in \mathcal{B}(v)\}
\end{aligned}
$$

There must be at least one real root $u \in \mathcal{B}(v), \forall v \in \mathcal{A}$, otherwise Assumption 1 would not be satisfied. We can now use the implicit function theorem. For all pairs of controls $(v, u) \in \mathcal{C}$ the equation (10) holds. If for every $v \in \mathcal{A}$ there exists at least one $u \in \mathcal{B}(v)$ for which:

$$
\left.\frac{\partial f}{\partial u}\right|_{(v, u)} \neq 0
$$

then the implicit function theorem guarantees the existence of a function $u=g(0, v)$, which is $C^{\infty}$ since we deal with polynomials, such that $f(0, v, g(0, v))=0$.

The implicit function theorem gives only sufficient conditions to check Lemma 5.3 but they are easy to check. If (24) does not hold, we may check whether Lemma 5.3 is satisfied. The easiest way to do this is to draw the variety $V_{z}$ around every point $(v, u)$ in $\mathcal{C}$ using Matlab (the set $\mathcal{C}$ contains finitely many points) and check whether there exists a branch of $V_{z}$ which does not have a vertical asymptote at $(v, u)$.

Before we give the classification of all possible situations we define bisectors and octants.

$$
\begin{gathered}
B_{1}=\left\{(v, u) \in \Re^{2}: v=u\right\}, \quad B_{2}=\left\{(v, u) \in \Re^{2}:-v=u\right\} \\
O_{1}=\left\{(v, u) \in \Re^{2}: v>0, u>0, u<v\right\}, O_{2}=\left\{(v, u) \in \Re^{2}: v>0, u>0, u>v\right\} \\
O_{3}=\left\{(v, u) \in \Re^{2}: v<0, u>0, u>-v\right\}, O_{4}=\left\{(v, u) \in \Re^{2}: v<0, u>0, u<-v\right\} \\
O_{5}=\left\{(v, u) \in \Re^{2}: v<0, u<0, u>v\right\}, O_{6}=\left\{(v, u) \in \Re^{2}: v<0, u<0, u<v\right\} \\
O_{7}=\left\{(v, u) \in \Re^{2}: v>0, u<0, u<-v\right\}, O_{8}=\left\{(v, u) \in \Re^{2}: v>0, u<0, u>-v\right\}
\end{gathered}
$$

We also use notation $A_{1}$ and $A_{2}$ to denote respectively the line $v=0$ and $u=0$ in $\Re^{2}$.

A very important concept of the "inverse graph" of the variety $V_{z}(14)$ which is given by:

$$
V_{z}^{-1}=\left\{(v, u) \in \Re^{2}: f(0, u, v)=0\right\}
$$

is obtained by simply interchanging variables $v$ and $u$ in the defining polynomial. It is easy to check that if a point on a variety $V_{z}$ is in the first octant $O_{1}$, the corresponding point on $V_{z}^{-1}$ is in the second octant $O_{2}$ and vice versa. We use the following notation to summarise all possible situations:

$$
O_{2} \leftrightarrow O_{1}, O_{3} \leftrightarrow O_{8}, O_{4} \leftrightarrow O_{7}, O_{5} \leftrightarrow O_{6}
$$


In some cases the position of branches $V_{M}^{S_{2}}$ and $V_{m}^{S_{1}}$ provide sufficient information to conclude on the stability of constrained dynamics since the conditions on the inverse graph are automatically satisfied. We summarise these trivial cases in the Lemma below.

LEMMA 6.1.

1. If one of the following conditions hold

(a) $V_{m}^{S_{1}} \subset O_{5}$ and $V_{M}^{S_{2}} \subset O_{1}$

(b) $V_{m}^{S_{1}} \subset O_{5}$ and $V_{M}^{S_{2}} \subset O_{8}$

(c) $V_{m}^{S_{1}} \subset O_{5}$ and $V_{M}^{S_{2}} \subset O_{7}$

(d) $V_{m}^{S_{1}} \subset O_{4}$ and $V_{M}^{S_{2}} \subset O_{1}$

(e) $V_{m}^{S_{1}} \subset O_{4}$ and $V_{M}^{S_{2}} \subset O_{8}$

(f) $V_{m}^{S_{1}} \subset O_{3}$ and $V_{M}^{S_{2}} \subset O_{1}$

then there exist a criterion of choice which yields stable constrained dynamics.

2. If $V_{m}^{S_{1}} \subset B_{2}\left(V_{M}^{S_{2}} \subset B_{2}\right)$ then there exists a criterion of choice which yields stable constrained dynamics if and only if $V_{M}^{S_{2}}\left(V_{m}^{S_{1}}\right)$ belongs to the cone $\left\{(v, u) \in \Re^{2}:|v|<|u|\right\}$.

3. If $V_{m}^{S_{1}} \subset A_{2}$ or $V_{M}^{S_{2}} \subset A_{2}$, the constrained dynamics are stable.

4. If $V_{m}^{S_{1}}=\emptyset$ or $V_{M}^{S_{2}}=\emptyset$ or $V_{m}^{S_{1}}=\emptyset$ and $V_{M}^{S_{2}}=\emptyset$ then the constrained dynamics are unstable.

5. If $V_{m}^{S_{1}} \subset O_{3}$ or $V_{M}^{S_{2}} \subset O_{7}$ or $V_{m}^{S_{1}} \subset O_{3}$ and $V_{M}^{S_{2}} \subset O_{7}$ then the constrained dynamics are unstable.

It can easily be checked that the only remaining cases are:

1. $V_{m}^{S_{1}} \subset O_{3}$ and $V_{M}^{S_{2}} \subset O_{8}$

2. $V_{m}^{S_{1}} \subset O_{4}$ and $V_{M}^{S_{2}} \subset O_{7}$

Only in these cases do we have to use "inverses" $\left(V_{m}^{S_{1}}\right)^{-1}$ and $\left(V_{M}^{S_{2}}\right)^{-1}$. Since we are dealing with polynomial systems, we can use the algebraic structure of these systems in order to obtain a "box" inside which all intersections between $V_{z}$ and $V_{z}^{-1}$ occur (modulo common components). We will use the theory of resultants to compute such a box. We denote $f_{1}=f(0, v, u)$ and $f_{2}=f(0, u, v)$.

Resultants procedure:

First, we find the greatest common divisor of $f_{1}$ and $f_{2}$ which is denoted as $G C D\left(f_{1}, f_{2}\right) \in \Re[v, u]$. Then we compute "common components free" polynomials:

$$
f_{1}^{c c f}=\frac{f_{1}}{G C D\left(f_{1}, f_{2}\right)} \quad f_{2}^{c c f}=\frac{f_{2}}{G C D\left(f_{1}, f_{2}\right)}
$$

Now, we can regard polynomials $f_{1}^{c c f}$ and $f_{2}^{c c f}$ as polynomials in $v$ whose coefficients are polynomials in $u$. Now we can find the resultant of the two polynomials:

$$
R\left(f_{1}^{c c f}, f_{2}^{c c f}\right)=\sum_{i=0}^{p} a_{i} u^{i}
$$

The resultant $R\left(f_{1}^{c c f}, f_{2}^{c c f}\right)$ is a polynomial in $u$. We know that polynomials $f_{1}^{c c f}$ and $f_{2}^{c c f}$ have no common roots if $R\left(f_{1}^{c c f}, f_{2}^{c c f}\right) \neq 0$. We can find a number $D_{2}$ which is such that all absolute values of real roots of the resultant are less than $D_{2}$.

Second, we estimate the number $D_{2}$ using formulas for bounds on roots, e.g. $\hat{D}_{2}=1+\sup _{i}\left|a_{i}\right|$ [4], where $a_{i}, i=0,1, \ldots, p$ are coefficients of the resultant. Outside the box defined by $\left\{(v, u) \in \Re^{2}:|v| \leq \hat{D}_{2}\right.$ and $\left.|u| \leq \hat{D}_{2}\right\}$ the varieties $V_{z}$ and $V_{z}^{-1}$ have no intersections modulo common branches. 
Third, we pick $\hat{u}$ such that $|\hat{u}|>\left|\hat{D}_{2}\right|$ and find sets of solutions:

$$
\Sigma_{1}=\{v \in \Re: f(0, v, \hat{u})=0\}, \quad \Sigma_{2}=\{v \in \Re: f(0, \hat{u}, v)=0\}
$$

We can see that the sets $\Sigma_{1}$ and $\Sigma_{2}$ give a complete picture about the branches of varieties $V_{z}$ and $V_{z}^{-1}$ and therefore can be used to check whether constrained dynamics are stable for the two remaining cases. The criterion for the stability of constrained dynamics of the two last cases, which are not covered by Lemma 6.1 , is given in the following Lemma.

LEMMA 6.2. If

1. $V_{m}^{S_{1}} \subset O_{3}$ and $V_{M}^{S_{2}} \subset O_{8}$ or

2. $V_{m}^{S_{1}} \subset O_{4}$ and $V_{M}^{S_{2}} \subset O_{7}$

then constrained dynamics are stable if there exist $\sigma_{1} \in \Sigma_{1}$ and $\sigma_{2} \in \Sigma_{2}$ such that $\sigma_{1}<\sigma_{2}$. In the first case sets $\Sigma_{1}$ and $\Sigma_{2}$ (28) are calculated using $\hat{u}>\hat{T}$ and in the second case $\hat{u}<-\hat{T}$.

Proof of Lemma 6.2: It trivially follows from Theorem 5.4 and the above given procedure.

The method to check the existence of constrained dynamics consists of several steps:

1. Check the conditions of Lemma 5.3 as described before.

2. Form the Sturm sequence and find all leading coefficient functions. Using (17) and bounds on roots, determine the estimate $\hat{D}_{1}$.

3. Find the box inside which all intersections between the variety $V_{z}$ and $B_{1}, B_{2}, A_{1}$ and $A_{2}$ occur. This is done in the following way. Find the following estimates:

$\hat{D}_{3}=1+\max _{i}\left|n_{i}\right|, \hat{D}_{4}=1+\max _{i}\left|m_{i}\right|, \hat{D}_{5}=1+\max _{i}\left|k_{i}\right|, \hat{D}_{6}=1+\max _{i}\left|l_{i}\right|$

where $n_{i}, m_{i}, k_{i}, l_{i} \in \Re$ are respectively coefficients of polynomials $f(0, v, v)$, $f(0, v,-v), f(0,0, u)$ and $f(0, v, 0)$.

4. Find the estimate $\hat{T}$ of $T$ using:

$$
\hat{T}=\max \left(\hat{D}_{1}, \hat{D}_{3}, \hat{D}_{4}, \hat{D}_{5}, \hat{D}_{6}\right)
$$

5. Pick any $\left.v^{*} \in\right]-\infty,-\hat{T}[$ and compute all real roots of

$$
f\left(0, v^{*}, u\right)=0
$$

Pick any $\left.v^{* *} \in\right] \hat{T},+\infty[$ and compute all real roots of

$$
f\left(0, v^{* *}, u\right)=0
$$

6. Determine to which octants do the pairs $\left(v^{*}\right.$, real root to $\left.(30)\right)$ and $\left(v^{* *}\right.$, real root to (31)) belong and check whether Lemma 6.1 holds (remember that checking the position of a single point of the variety implies that the whole branch has the same position). If Lemma 6.1 is not satisfied then proceed onto the next step.

7. Compute $\hat{D}_{2}=1+\max _{i}\left|f_{i}\right|$ where $f_{i}$ are the coefficients of the resultant $R\left(f_{1}^{c c f}, f_{2}^{c c f}\right)$, redefine $\hat{T}=\max \left(\hat{D}_{1}, \hat{D}_{2}, \hat{D}_{3}, \hat{D}_{4}, \hat{D}_{5}, \hat{D}_{6}\right)$ and apply the resultants procedure which is used to check conditions of Lemma 6.2. 


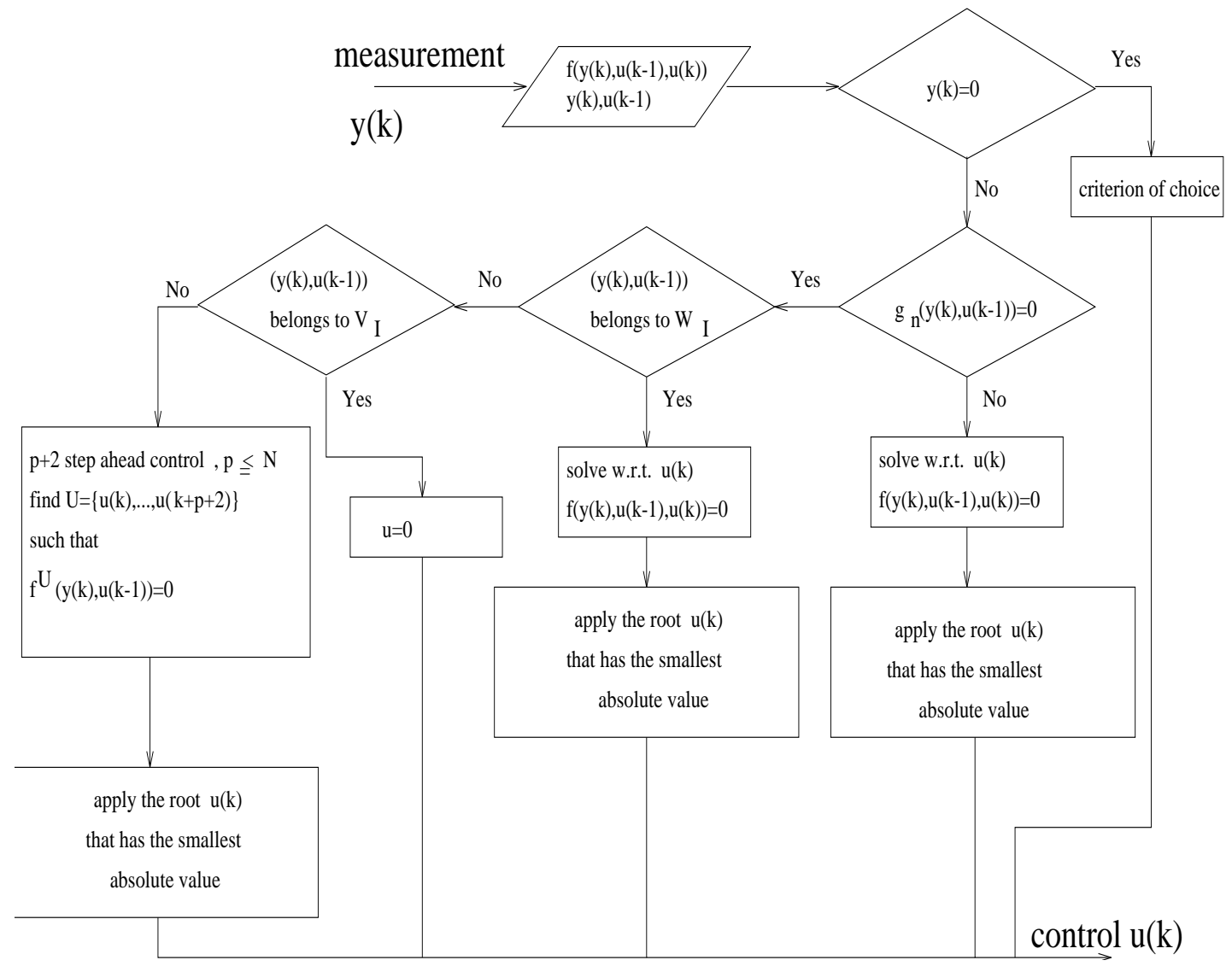

FIG. 1. Output dead beat controller - algorithm

7. Output Dead Beat Control Law with Stable Constrained Dynamics. Propositions 4.1-4.5 can be used to design a dead beat controller (algorithm) as outlined in Figure 1. The obtained controller uses static feedback to compute the value of control signal at any time instant $k$. The closed loop system can be written in the form:

$$
\begin{array}{r}
y_{k+1}=f\left(y_{k}, u_{k-1}, u_{k}\right) \\
u_{k}=c\left(y_{k}, u_{k-1}\right)
\end{array}
$$

The control signal is obtained as a solution to a polynomial algebraic equation and since there may be more than one solution we need a criterion of choice to define the control law $c\left(y_{k}, u_{k-1}\right)$. One criterion for the choice may be: apply the control signal that has the least absolute value. We may be able to shape the transient response and keep the control signals as small as feasible, using a different criterion of choice. The question of which choice is not so critical if the output is not zero. Having zeroed the output, the criterion of choice becomes crucial for the stability of constrained dynamics and, consequently, for the stability of the closed loop system (32). 
A criterion of choice which yields stable constrained dynamics is given by:

$$
u_{k}= \begin{cases}u \in V_{m}^{S_{1}} & \text { if }(v, u) \in S_{1} \\ u \in V_{M}^{S_{2}} & \text { if }(v, u) \in S_{2} \\ u \text { s.t. it has minimum absolute value } & \text { if } v \in[-\hat{T}, \hat{T}]\end{cases}
$$

This choice does not guarantee the fastest convergence to the invariant interval and other choices may be better in this sense than this control law. The tradeoff between the speed of convergence to the invariant interval and the shape of the transient response is a difficult problem in its own right but very often it is possible to successfully tackle this problem on a case by case basis.

Notice that working with poor bounds on roots, such as the one that we have used, may yield an estimate $\hat{T}$ which is much larger than the minimal possible $T$, but the computations are simpler and faster to use when checking the existence of stable constrained dynamics. Computing exact roots, on the other hand, yields a smaller size of the invariant interval, which should be used when implementing the controller. Blocks in which we need to check whether $(y(k), u(k-1))$ belong to $W_{I}$ or $V_{I}$ are equivalent to testing whether a finite number of polynomials which define $W_{I}$ and $V_{I}$ are zero when evaluated at $(y(k), u(k-1))$.

8. Examples. The following example illustrates the concepts of invariant and strongly invariant subsets of the variety $V_{C}$.

EXAMPLE 1. Consider the system:

$$
y_{k+1}=\left(y_{k}-u_{k-1}^{2}-1\right)\left(y_{k}+u_{k-1}^{2}+1\right)\left[\left(y_{k}+2\right) u_{k}^{3}+u_{k}^{2}+1\right]+u_{k}^{2}+1
$$

Assumption 1 is satisfied. The critical variety $V_{C}$ is defined by:

$$
V_{C}=\left\{(y, v) \in \Re^{2}:\left(y-v^{2}-1\right)\left(y+v^{2}+1\right)(y+2)=0\right\}
$$

In this case we can verify that the only strongly invariant set is given by:

$$
W_{I}=\left\{(y, v) \in \Re^{2}:\left(y-v^{2}-1\right)=0\right\} \subset V_{C}
$$

We check the existence of strongly invariant sets via Proposition 4.5. There are three varieties of Special Form that are contained in $V_{C}$

$$
y-v^{2}-1 ; \quad y+v^{2}+1 ; \quad y+2
$$

and we also have

$$
g_{0}=\left(y-v^{2}-1\right)\left(y+v^{2}+1\right)+1 ; \quad g_{1}=0 ; \quad g_{2}=\left(y-v^{2}-1\right)\left(y+v^{2}+1\right)+1
$$

The only cycle of Proposition 4.5 is given by the divisions:

$$
g_{0} \equiv 1\left|\left(y-v^{2}-1\right) ; \quad g_{1} \equiv 0\right|\left(y-v^{2}-1\right) ; \quad g_{2} \equiv 1 \mid\left(y-v^{2}-1\right)
$$

which defines $W_{I}$. Since $W_{I}$ does not intersect the line $y=0$ according to Theorem 4.6 the system is not output dead beat controllable.

We have, therefore, $W_{I} \stackrel{f}{\rightarrow} W_{I}$, and $t$ in Definition 8 can be chosen to be 1 . From equation (34) it is clear that $\forall(y, v) \in V_{1}$, where $V_{1}=\left\{(y, v) \in \Re^{2}:\left(y+v^{2}+1\right)=0\right\}$ (see Figure 2) we have $V_{r}(y, v)=W_{I}$. Therefore, any initial state in $V_{1}$ is transferred 


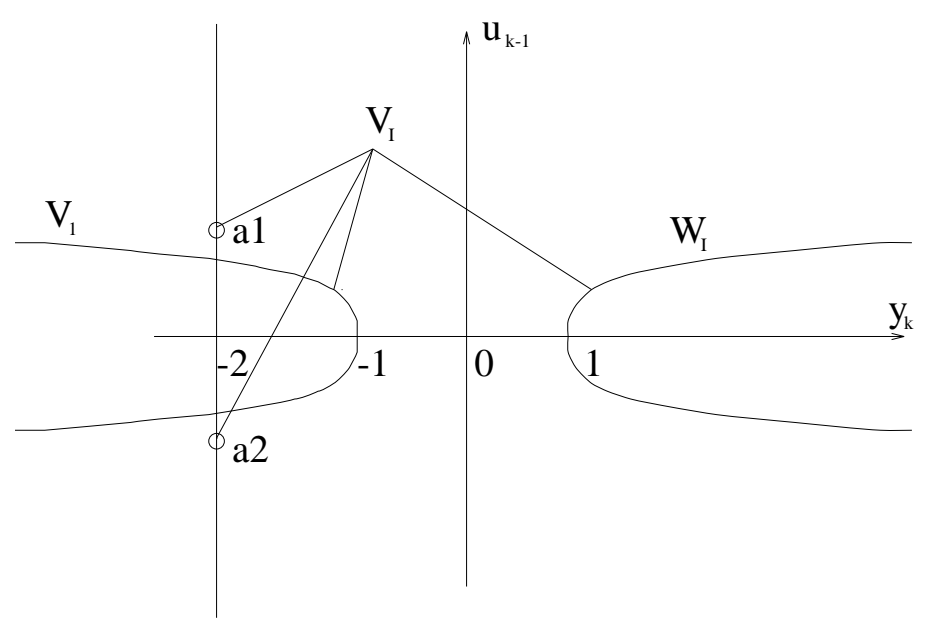

FIG. 2. Invariant sets $V_{I}$ and strongly invariant sets $W_{I}$ (Example 1)

in one step to some point in $W_{I}$ irrespective of the control that is applied. Thus, we can write:

$$
V_{1} \stackrel{f}{\rightarrow} W_{I} \stackrel{f}{\rightarrow} W_{I} \stackrel{f}{\rightarrow} \ldots
$$

Consider now initial states on the line $y_{0}=-2$. The model of the system becomes:

$$
y_{1}=\left[\left(-3-u_{-1}^{2}\right)\left(-1+u_{-1}^{2}\right)+1\right]\left(u_{0}^{2}+1\right) .
$$

Denote real solutions $u_{-1}$ of the following equations:

$$
\begin{gathered}
{\left[\left(-3-u_{-1}^{2}\right)\left(-1+u_{-1}^{2}\right)+1\right]=-1} \\
{\left[\left(-3-u_{-1}^{2}\right)\left(-1+u_{-1}^{2}\right)+1\right]=1}
\end{gathered}
$$

as $a_{i}$ and $b_{i}(i=1,2)$, respectively. The set of one step reachable states from $\left(-2, a_{1}\right)$ and $\left(-2, a_{2}\right)$ is $V_{1}$ and from $\left(-2, b_{1}\right)$ and $\left(-2, b_{2}\right)$ is $W_{I}$. Notice also that $b_{1}=1, b_{2}=$ -1 and hence $\left(-2, b_{1}\right)$ and $\left(-2, b_{2}\right)$ belong to $V_{1}$. Therefore, we can write:

$$
\left(-2, a_{i}\right) \stackrel{f}{\rightarrow} V_{1} \stackrel{f}{\rightarrow} W_{I} \stackrel{f}{\rightarrow} W_{I} \stackrel{f}{\rightarrow} \ldots, i=1,2
$$

The maximal invariant set $V_{I}$ is: $V_{I}=\left\{(y, v) \in \Re^{2}:\left(y-v^{2}-1\right)\left(y+v^{2}+1\right)=0\right\} \cup\left\{\left(-2, a_{1}\right),\left(-2, a_{2}\right)\right\}$.

Sets $V_{I}$ and $W_{I}$ are shown in Figure 2. The set $V_{C}-V_{I}$ is not invariant and there exists a control $u_{k}$ which can map any initial state from it to $\Re^{2}-V_{C}$ in one step. Observe that both $V_{I}$ and $W_{I}$ are real varieties, whereas $V_{C}-V_{I}$ is not. Also, initial states in $V_{1}$ are transferred to $W_{I}$ in one step and the initial states $\left(-2, a_{i}\right), i=1,2$ are transferred to $W_{I}$ in two steps.

The following example serves to illustrate why the present notion of stability of constrained dynamics is more appropriate in this context than the notion of zero dynamics introduced in $[17,18]$. 
ExAmple 2. Consider the following system:

$$
y_{k+1}=\left(u_{k}+2 u_{k-1}+y_{k}\right)\left(u_{k}-0.5 u_{k-1}-y_{k}\right)
$$

We introduce the state variables $x_{1}(k)=y_{k}$ and $x_{2}(k)=u_{k-1}$ and write:

$$
\begin{aligned}
x_{1}(k+1) & =\left(u_{k}+2 x_{2}(k)+x_{1}(k)\right)\left(u_{k}-0.5 x_{2}(k)-x_{1}(k)\right) \\
x_{2}(k+1) & =u_{k} \\
y(k) & =x_{1}(k)
\end{aligned}
$$

According to [17], the relative degree for system (36) is $d=1$ and Assumption 1 in [17] holds. Two possible feedback laws can be used to transform the system into the form (2.6) in [17]:

$$
\begin{aligned}
& u_{k}=\frac{-1.5 x_{2}(k)+\sqrt{6.25 x_{2}^{2}(k)+10 x_{1}(k) x_{2}(k)+4 x_{1}^{2}(k)+4 v(k)}}{2} \\
& u_{k}=\frac{-1.5 x_{2}(k)-\sqrt{6.25 x_{2}^{2}(k)+10 x_{1}(k) x_{2}(k)+4 x_{1}^{2}(k)+4 v(k)}}{2},
\end{aligned}
$$

where $v(k)$ is the new control input. If we use the control law (37), the the corresponding zero dynamics are then defined as $x_{2}(k+1)=-2 x_{2}(k)$ (with $\left.x_{1}(k)=0, v(k)=0\right)$ and are obviously not stable. If, on the other hand, we had chosen (36), we obtain $x_{2}(k+1)=0.5 x_{2}(k)$, which is obviously stable. In this case there are 4 different continuous feedback laws that transform the system into the form (2.6) in [17]. Three of them yield stable zero dynamics and one yields unstable zero dynamics. Also, there are infinitely many discontinuous control laws that keep the output at zero. Notice, that all conditions in [17] are satisfied and it appears that the stability of the zero dynamics depends on the choice of the feedback law. The criterion of choice that we use in the definition of stable constrained dynamics takes this phenomenon explicitly into account.

The following example illustrates the method for checking the existence of stable constrained dynamics.

ExAmPle 3. Check the existence of stable constrained dynamics for the following system:

$y_{k+1}=-2\left(1+y_{k}^{2}\right) u_{k}^{5}-2 u_{k}^{3}+2 u_{k} u_{k-1}\left(1+y_{k}^{4}\right)+2 u_{k} u_{k-1}^{2}+u_{k-1} u_{k}^{4}+u_{k-1} u_{k}^{2}-u_{k-1}^{2}-u_{k-1}^{3}+y_{k}^{3}$

For $y_{k}=0$ we have:

(38) $-2 u_{k}^{5}-2 u_{k}^{3}+2 u_{k} u_{k-1}+2 u_{k} u_{k-1}^{2}+u_{k-1} u_{k}^{4}+u_{k-1} u_{k}^{2}-u_{k-1}^{2}-u_{k-1}^{3}=0$

Therefore, the variety $V_{z}$ is defined by:

$$
V_{z}=\left\{(v, u) \in \Re^{2}:-2 u^{5}-2 u^{3}+2 u v+2 u v^{2}+v u^{4}+v u^{2}-v^{2}-v^{3}=0\right\} .
$$

We will follow the steps that are described in Section 6 in order to check the existence of stable constrained dynamics.

Step 1: Since $g_{5}(0, v)=-2$ the conditions of Lemma 5.3 are satisfied. 
Step 2: Using Maple ${ }^{3}$, we obtain the following Sturm sequence:

$$
\begin{aligned}
f_{0}= & -2 u^{5}-2 u^{3}+2 u v+2 u v^{2}+v u^{4}+v u^{2}-v^{2}-v^{3} \\
f_{1}= & -10 u^{4}-6 u^{2}+2 v+2 v^{2}+4 v u^{3}+2 v u \\
f_{2}= & -\left(-\frac{4}{5}+\frac{2}{25} v^{2}\right) u^{3}-\frac{12}{25} v u^{2}-\left(\frac{41}{25} v^{2}+\frac{8}{5} v\right) u+\frac{24}{25}\left(v^{2}+v^{3}\right) \\
f_{3}= & -25 \frac{\left(-24+7 v^{4}+8 v^{3}-80 v-82 v^{2}\right) u^{2}}{\left(-10+v^{2}\right)^{2}}+50 \frac{v\left(-15 v^{2}+4 v^{3}+4 v^{4}-16 v-4\right) u}{\left(-10+v^{2}\right)^{2}} \\
& -50 \frac{4 v^{3}+v^{4}+v^{5}+4+4 v+4 v^{2}}{\left(-10+v^{2}\right)^{2}} \\
f_{4}= & -\left[8 \left(12800 v+41680 v^{2}+68240 v^{3}+52516 v^{4}+7268 v^{5}-10960 v^{6}\right.\right. \\
& \left.-3152 v^{7}+449 v^{8}+133 v^{9}+8 v^{10}+4 v^{11}+1600\right] /\left[2 5 \left(-24+7 v^{4}\right.\right. \\
& \left.\left.+8 v^{3}-80 v-82 v^{2}\right)^{2}\right]+\left[v \left(161600 v+548160 v^{2}+923680 v^{3}\right.\right. \\
& +727392 v^{4}+113716 v^{5}-142400 v^{6}-41100 v^{7}+4456 v^{8}+1033 v^{9} \\
& \left.\left.+196 v^{10}+100 v^{11}+19200\right) u\right] /\left[25\left(-24+7 v^{4}+8 v^{3}-80 v-82 v^{2}\right)^{2}\right] \\
= & {\left[5 0 \left(49 v^{15}+161 v^{14}-2148 v^{13}-8948 v^{12}+27908 v^{11}+175332 v^{10}\right.\right.} \\
& +5760 v^{9}-1338048 v^{8}-2333952 v^{7}+1619072 v^{6}+10299904 v^{5}+15313920 v^{4} \\
& \left.\left.+11967488 v^{3}+5407744 v^{2}+1351680 v+147456\right) v\right] /\left[\left(25 v^{5}+24 v^{4}\right.\right. \\
& \left.\left.+728 v^{3}+1360 v^{2}+848 v+192\right)^{2}\left(-10+v^{2}\right)^{2}\right]
\end{aligned}
$$

From the Sturm sequence we find the leading coefficient functions:

$$
\begin{aligned}
(40)+ & \left.\left.+196 v^{10}+100 v^{11}+19200\right)\right] /\left[25\left(-24+7 v^{4}+8 v^{3}-80 v-82 v^{2}\right)^{2}\right], \\
& {\left[5 0 \left(49 v^{15}+161 v^{14}-2148 v^{13}-8948 v^{12}+27908 v^{11}+175332 v^{10}\right.\right.} \\
& +5760 v^{9}-1338048 v^{8}-2333952 v^{7}+1619072 v^{6}+10299904 v^{5}+15313920 v^{4} \\
& \left.\left.+11967488 v^{3}+5407744 v^{2}+1351680 v+147456\right) v\right] /\left[\left(25 v^{5}+24 v^{4}\right.\right. \\
& \left.\left.+728 v^{3}+1360 v^{2}+848 v+192\right)^{2}\left(-10+v^{2}\right)^{2}\right]
\end{aligned}
$$$$
-2,-10,-\left(-\frac{4}{5}+\frac{2}{25} v^{2}\right)
$$$$
-25 \frac{\left(-24+7 v^{4}+8 v^{3}-80 v-82 v^{2}\right)}{\left(-10+v^{2}\right)^{2}},
$$$$
\left[v \left(161600 v+548160 v^{2}+923680 v^{3}+727392 v^{4}+113716 v^{5}\right.\right.
$$$$
-142400 v^{6}-41100 v^{7}+4456 v^{8}+1033 v^{9}
$$

Using the formula for bounds on roots [4] we find that the highest coefficient functions do not change their signs for $v$ belonging to intervals ] $-\infty,-312529.98$ [ and ] $312529.98,+\infty\left[\right.$. In other words, the estimate of $D_{1}$ is $\hat{D}_{1}=312529.98$.

Step 3: All intersections of the variety $V_{z}$ with $A_{1}, A_{2}, B_{1}$ and $B_{2}$ lay in the interval ] $-4,+4\left[\right.$. It is easy to check that $\hat{D}_{3}=2, \hat{D}_{4}=4, \hat{D}_{5}=2$ and $\hat{D}_{6}=3$.

Step 4: Therefore, the estimates of sets $S_{1}$ and $S_{2}$ are defined using the number $\hat{T}=312529.98$.

Step 5: We now substitute any number $v$ from the interval ] $-\infty,-312529.98[$ into (38) and find all real roots. We obtain the following set of points in $\Re^{2}$ :

$$
\{(-312530, u):(-312530,+559.04293),(-312530,-559.04293),(-312530,-156265)\}
$$

\footnotetext{
${ }^{3}$ Copyright (c) 1981-1992 by the University of Waterloo
} 
Similarly, we obtain the set of roots

$$
\{(312530, u):(+312530,559.04383),(312530,-559.04383),(312530,156265)\}
$$

when we substitute $v^{* *}=312530$ that belongs to the interval $] 312529.98,+\infty[$ into (38). All these points represent branches and hence $V_{m}^{S_{1}} \subset O_{5}$ and $V_{M}^{S_{2}} \subset O_{1}$.

Step 6: We conclude that there exists stable constrained dynamics for this system since point 1.a of Lemma 6.1 is satisfied. We could work with better bounds on the roots in order to obtain better estimates for the intervals $S_{1}$ and $S_{2}$ or better still find the exact roots of the polynomials in the Sturm sequence. However, the proposed method is able to check the existence of the constrained dynamics quickly.

We have provided a constructive method to verify the existence of a criterion of choice leading to (globally) stable constrained dynamics. The method of $[17,18]$ appears not to be able to deal with this aspect in general, as the example shows. Indeed, the feedback law required in the method of $[17,18]$ for this example can not be expressed in an explicit form (this requires an analytic solution for a 5 th degree polynomial equation).

\section{REFERENCES}

[1] G.Bastin, F.Jarachi and I.M.Y.Mareels,Dead beat control of recursive nonlinear systems, Proc. 32nd IEEE Conference on Decision and Control, San Antonio, Texas, 1993, pp. 2965-2971.

[2] F.Jarachi, G.Bastin and I.M.Y.Mareels, One-step ahead control of nonlinear discrete time systems with one dimensional zero dynamics: global stability conditions, Proc. NOLCOS-III, Tahoë, USA, 1995.

[3] G.Bastin, F.Jarachi and I.M.Y.Mareels, Output deadbeat control of nonlinear systems in one step: feasibility and stability conditions, submitted for publication, 1994.

[4] R.Benedetti and J.J.Risler, Real algebraic and semi-algebraic sets, Hermann, 1990.

[5] S.A.Billings and W.S.F.Voon, A prediction-error and stepwise-regression estimation algorithm for non-linear systems, Int. J. Control, 44 (1986), pp.803-822.

[6] D.Cox, J. Little and D. O'Shea, Ideals, varieties and Algorithms, Springer-Verlag, 1992.

[7] M.E.Evans and D.N.P.Murthy, Controllability of a class of discrete time bilinear systems, IEEE Trans. Aut. Contr., 22 (1977), pp. 78-83.

[8] M.E.Evans and D.N.P.Murthy, Controllability of disrete time inhomogeneous bilinear systems, Automatica, 14 (1978), pp. 147-151.

[9] S.T.Glad, Output dead-beat control for nonlinear systems with one zero at infinity, Systems and Control Letters, 9 (1987), pp. 249-255.

[10] S.T.Glad, Dead beat control for nonlinear systems, in Analysis and control of nonlinear systems, C.I.Byrnes, C.F.Martin and R.E.Saeks, eds., North-Holland, 1988, pp. 437-442.

[11] T.Goka, T.J.Tarn and J.Zaborszky, On the controllability of a class of discrete bilinear systems, Automatica, 9 (1973), pp. 615-622.

[12] O.M.Grasselli, A.Isidori and F.Nicolo, Dead-beat control of discrete-time bilinear systems, International Journal of Control, 32 (1980), pp. 31-39.

[13] R.Haber and H.Unbehauen, Structure identification of nonlinear dynamic systems-a survey of input/output approaches, Automatica, 26 (1990), pp. 651-677.

[14] R.Haber and L.Keviczky, Identification of nonlinear dynamic systems, Proc. 4th IFAC Symp. on Identification and System Parameter Estimation, Tbilisi, USSR, 1978, pp. 79-126.

[15] V.L.Kocic and G.Ladas, Global behaviour of nonlinear difference equations of higher order with applications, Kluwer Academic Publishers, Dordrecht, 1993.

[16] J.O'Reilly, The discrete linear time invariant time-optimal control problem-an overview, Automatica, 17 (1981), pp. 363-370.

[17] S.Monaco and D.Normand-Cyrot, Minimum-phase nonlinear discrete-time systems, Proc. 28th C.D.C., Los Angeles, California, 1987, pp. 979-986. 
[18] S.Monaco and D.Normand-Cyrot, Zero dynamics of sampled nonlinear systems, Syst. Contr. Lett., 11 (1988), pp. 229-234.

[19] N.Rouche, P.Habets and M.Laloy, Stability theory by Liapunov's direct method, Springer-Verlag, Berlin, 1977.

[20] D.Nešić, I.M.Y.Mareels, R.Mahony and G.Bastin, $\nu$-step controllability of polynomial scalar systems, Proc. 3rd ECCC, Rome, Italy, 1994, pp. 277-282.

[21] D.Nešić, I.M.Y.Mareels, G.Bastin and R.Mahony, Necessary and sufficient conditions for output dead beat controllability for a class of polynomial systems, Proc. 34th CDC, New Orleans, Lousiana, 1995, pp. 7-12.

22] E.D.Sontag, Polynomial response maps, Springer-Verlag, Berlin, 1979.

[23] E.D.Sontag and Y.Rouchaleau, On discrete-time polynomial systems, Nonlinear Analysis, Theory, Methods and Applications, 1 (1976), pp. 55-64. 